\title{
Topology-Based Flow-Oriented Adaptive Network Coding-Aware Routing Scheme for VANETS
}

\author{
Muhammad Azhar Iqbal $^{{ }^{*}}$, Bin Dai ${ }^{2}$, Muhammad Arshad Islam ${ }^{1}$, Muhammad Aleem ${ }^{1}$ and \\ Nguyen-Son $\mathrm{Vo}^{3}$ \\ ${ }^{1}$ Department of Computer Science, Capital University of Science and Technology, \\ Islamabad - PAKISTAN \\ [e-mail: azhar@cust.edu.pk, arshad.islam@cust.edu.pk, aleem@cust.edu.pk ] \\ ${ }^{2}$ School of Electronic Information and Communications, Huazhong University of Science and Technology, \\ Wuhan, P. R. CHINA \\ [e-mail: daibin@hust.edu.cn ] \\ ${ }^{3}$ Electrical and Electronics Engineering, Duy Tan University, \\ Da Nang, VIETNAM \\ [e-mail: vonguyenson@dtu.edu.vn] \\ *Corresponding author: Muhammad Azhar Iqbal
}

Received August 24, 2017; revised September 14, 2017; accepted January 2, 2018;

published May 31, 2018

\begin{abstract}
Information theory progression along with the advancements being made in the field of Vehicular Ad hoc NETworks (VANETs) supports the use of coding-aware opportunistic routing for efficient data forwarding. In this work, we propose and investigate an adaptive coding-aware routing scheme in a specific VANET scenario known as a vehicular platoon. Availability of coding opportunities may vary with time and therefore, the accurate identification of available coding opportunities at a specific time is a quite challenging task in the highly dynamic scenario of VANETs. In the proposed approach, while estimating the topology of the network at any time instance, a forwarding vehicle contemplates the composition of multiple unicast data flows to encode the correct data packets that can be decoded successfully at destinations. The results obtained by using OMNeT++ simulator reveal that higher throughput can be achieved with minimum possible packet transmissions through the proposed adaptive coding-aware routing approach. In addition, the proposed adaptive scheme outperforms static transmissions of the encoded packets in terms of coding gain, transmission percentage, and encoded packet transmission. To the best of our knowledge, the use of coding-aware opportunistic routing has not been exploited extensively in available literature with reference to its implications in VANETs.
\end{abstract}

Keywords: Vehicular Ad hoc NETworks (VANETs), Vehicular Platoon, Ad hoc Routing Protocols, Wireless Network Coding, Coding-Aware Routing Protocols 


\section{Introduction}

Immense technological progress in the field of networks and communications has made possible the Inter-Vehicle Communication (IVC) on roads and named as Vehicular Ad hoc NETworks (VANETs). The VANET is a special subset of Mobile Ad hoc NETworks (MANETs) in which vehicles equipped with wireless interfaces communicate to each other (known as Vehicle-to-Vehicle (V2V) or Car-to-Car (C2C) communication) as well as with fixed roadside equipment (known as Vehicle-to-Infrastructure (V2I), Car-to-Infrastructure (C2I) or Vehicle-to-Road (V2R) communications) on highways and city roads [1] [2] [3]. Over the years, the VANET has attracted both the automotive industry as well as the research community with an objective of Telematics promotion towards the establishment of the Intelligent Transportation System (ITS) for the provisioning of a range of applications/services to both on-road drivers and passengers. Moreover, in recent years, it has been observed that in order to deal with specific issues of traffic congestion, highway capacity, $\mathrm{CO}_{2}$ emission etc., platoon-based driving pattern (vehicular platoon) has been encouraged in comparison to individual driving [4] [5] [6]. The platoon-based driving pattern is a co-operative way of driving pattern in which a group of vehicles with common interests communicate with each other. The applications of vehicular platoon systems are beneficiary in various aspects such as 1) traffic flow optimization to reduced congestion 2) promotion of green traffic concept to decrease fuel consumption and 3) provisioning of infotainment services with efficient data delivery among vehicles within a platoon [7] (as shown in Fig. 1 (a)).

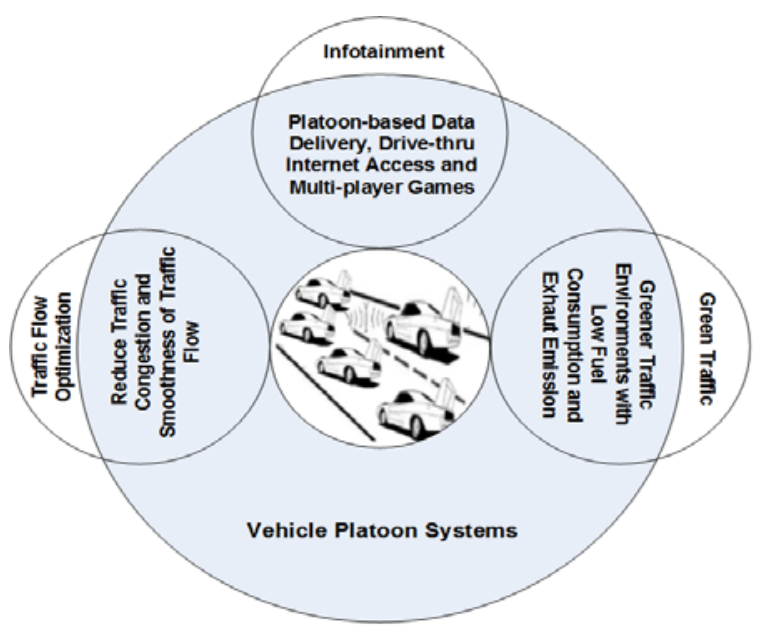

(a)

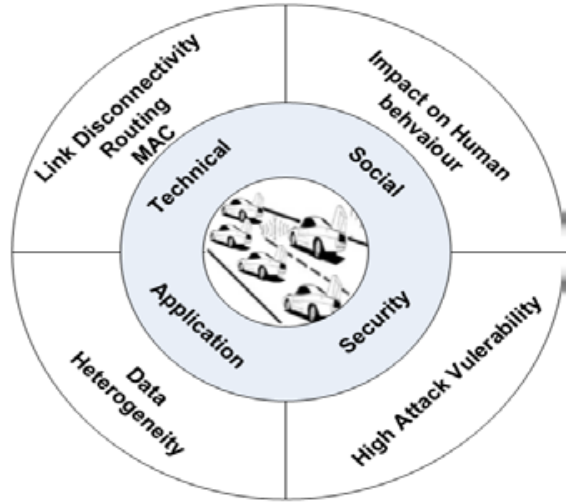

(b)

Fig. 1. (a) Types (b) Challenges of vehicular platoon systems

Efficient data dissemination in vehicular platoon demands resolution of arduous challenges such as technical (MAC, Routing), social (impact on human behavior), data heterogeneity, security/privacy etc. [8] [9] as summarized in Fig. 1 (b)). In this article, we have proposed an adaptive hop-by-hop coding-aware opportunistic routing scheme that performs inter-flow coding of data packets relying on the available information of the current network topology and the existing composition of multiple bidirectional unicast data flows at any instance of time. The proposed routing operation is validated through the simulations using OMNeT++ 
[10] [11]. The simulation-based analysis of the proposed approach demonstrates that appropriate encoding of data packets (belonging to different flows) eventually reduces the number of transmissions.

The rest of this paper is organized as follows: Section 2 provides detail about fundamental differences between coding-aware and coding-based routing protocols and associated challenges. Section 3 presents the proposed coding-aware routing scheme, which reduces the number of transmissions required for efficient data dissemination. Section 4 provides a mathematical model of the modified mobility model. The simulation modeling and analysis details for evaluation of the proposed approach are discussed in Section 5. Finally, we conclude this paper in Section 6.

\section{Opportunistic and Coding-Aware Routing}

In opportunistic routing, multiple neighbors can overhear a transmitting node and upon receiving a data packet, selected set of a sender's neighbor can continue the packet forwarding towards the destination while meeting the certain conditions [12] [13]. The contemporary opportunistic routing schemes can be categorized in various ways in terms of forwarder set selection and prioritization metric [14]. Regarding prioritization metric, Network Coding Based (NC-Based also known as Intra-flow) and Network Coding-Aware (NC-Aware also known as Inter-flow) approaches make forwarding decisions based on the available coding opportunities across several directions towards the destination node(s) within ad hoc networks [15]. To provide a better overview, the difference between NC-Based and NC-Aware routing (by depicting the phenomenon of mixing data packets) is elaborated in Fig. 2(a) and Fig. 2(b).

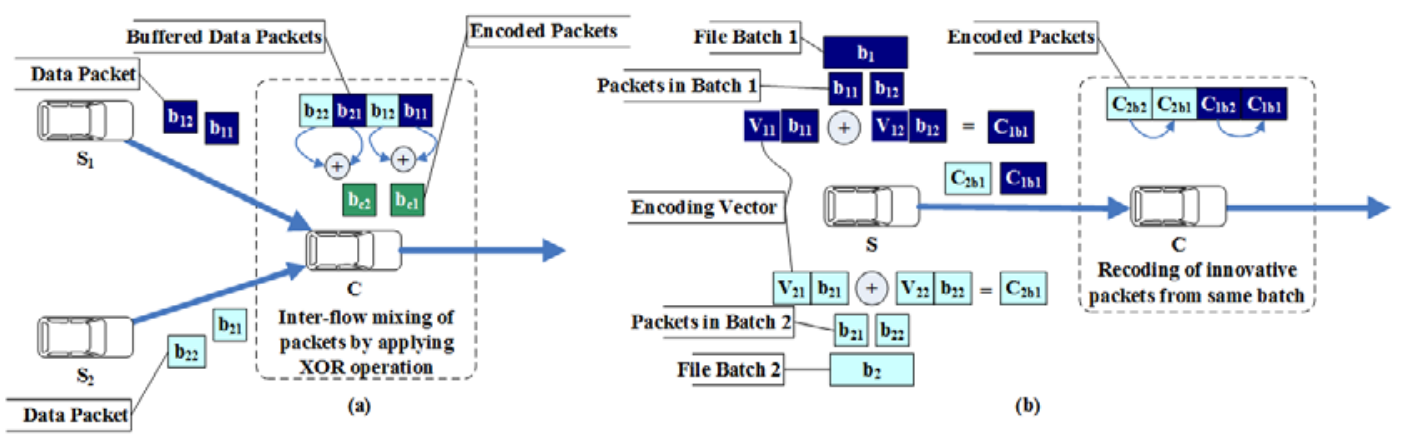

Fig. 2. (a) NC-Aware routing (b) NC-Based routing in wireless ad hoc networks

Fig. 2 (a) illustrates NC-Aware routing, where two vehicles $V_{1}$ and $V_{2}$ have packets for downstream vehicle $V_{C}$. Upon receiving the packets, the vehicle $V_{C}$ combines packets received from $V_{1}$ and $V_{2}$ and then forwards the combined packet to other downstream vehicle(s) that are able to decode and recover required data packet. Fig. 2 (b) illustrates the NC-based phenomenon, wherein the source vehicle $V$ broadcasts the linearly combined coded packets that belong to certain batches. Upon receiving, vehicle $V_{C}$ stores these innovative (linearly independent) encoded packets into its buffer and re-encodes (buffered packets of the same batch) before forwarding towards the destination vehicle.

The implication of coding-based data dissemination in VANETs has been extensively analyzed in the literature [16] [17] [18] [19] [20]. In coding-based techniques, relayed data from a single source is actualized as a combination of random linear network codes. To the best of our knowledge, the existing NC-Aware routing schemes have not been exploited in an extensive manner with reference to its implementation in VANETs. However, the existence of 
collaborative communication among vehicles within a specific stable platoon can take advantage of coding-aware opportunities. In this way, high throughput can be achieved with a reduced number of transmissions. In this research work, we have investigated the implementation challenges and associated advantages of the coding-aware routing for efficient data dissemination in the form of encoded data packets within a vehicular platoon.

\subsection{NC-Aware Routing Challenges in VANETs}

The implementation of NC-Aware routing in static wireless scenarios [21] [22] [23] [24] [25] [26] provides a fundamental basis to understand the usage of these routing schemes in dynamic ad hoc scenario. Following are the potential challenges of NC-Aware routing in dynamic VANETs:

- The accurate discovery of the available coding opportunities is dependent on the collection of reception reports that each vehicle receives from its neighboring vehicles;

- Availability of the coding opportunities depends on both the topology of the network and the composition of multiple bidirectional unicast data flows within the network. Therefore, knowledge of correct nodes for partial or full availability of encoding is required to be accurately known at each instance of time;

- The cost of NC-Aware due to the XOR operation at coding points is usually low. However, the waiting time for the arrival of data packets from different flows is also required to be low;

- The higher number of flows within a network can ultimately increase coding gain. However, in VANETs, NC-Aware may yield poor performance gain if waiting time of the buffered packet(s) is not adjusted appropriately. In other words, suitably fast encoding of the data packets is required at coding points in highly dynamic VANETs;

- VANETs may be metropolitan-scale networks; therefore, NC-Aware routing protocols should consider the appropriate level of scalability.

In addition to the above-mentioned challenges, we have considered general design goals (i.e., future event prediction, neighborhood discovery, multihop routing capability, and localized operations etc.) of VANET routing protocols. Fig. 3 illustrates encompassing general design goals that had been taken into account for the development of the various VANETs routing protocols.

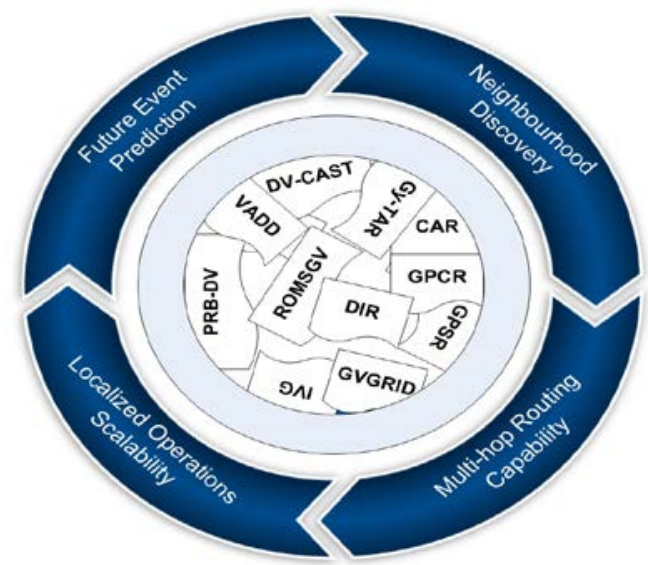

Fig. 3. VANETs routing protocols and associated general design goals 


\subsection{Limitations of the Existing NC-Aware Schemes in VANET}

In [14], the authors have recommended the taxonomy of NC-Aware routing approaches for ad hoc networks as shown in Fig. 4.

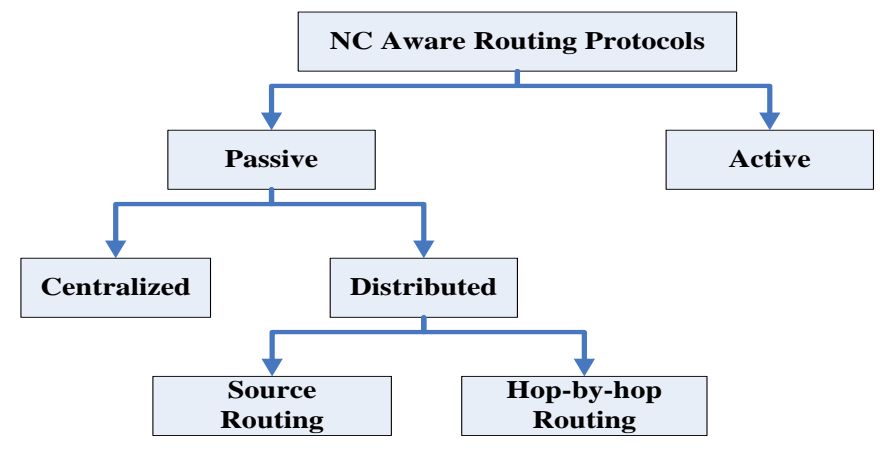

Fig. 4. Taxonomy of NC-Aware routing protocols for ad hoc networks [14]

Merits and demerits of the existing NC-Aware routing techniques with reference to their implications in VANETs are discussed below:

- In Active NC-Aware routing schemes, each node actively optimizes the routing of data packets along with the selected paths to exploit the highly available coding opportunities to reduce the number of transmissions. However, the initial steps related to the identification of routes from the source node(s) to the destination node(s) are same as source routing steps that ultimately limits the scope of active NC-Aware routing within VANETs.

- Centralized NC-Aware routing techniques are not suitable for VANETs because of sudden topology changes that may occur before the resolution of a central controller. Therefore, at any instance of time, it is difficult to find out the exact number of packets (to be coded) that could be decoded at destination nodes through centralized programming formulation schemes in dynamic scenarios.

- The nature of VANETs fundamentally supports the use of Distributed NC-Aware routing approaches, where each node is responsible to take routing decision locally. In vehicular scenarios, protocols that make routing decisions based on the current network state are more suitable than those protocols where routing decisions are based on already established network state. Therefore, in highly dynamic scenarios of VANETs, hop-by-hop NC-Aware routing schemes are more suitable than the source NC-Aware routing approaches. (In this work, the simulations are performed in OMNeT++ to compare and prove the effectiveness of hop-by-hop NC-Aware routing over source NC-Aware routing schemes).

\subsection{Source vs Hop-by-hop Coding-Aware Routing in VANETs}

Using OMNeT++, three simple scenarios are implemented. Following Freeway Mobility Model (FMM) [27], simulations are carried out for a 3-lane highway (depicting one side of a highway) with a length of $5 \mathrm{~km}$ and a width of $10 \mathrm{~m}$ per lane. In all simulations, having a transmission range of $250 \mathrm{~m}$, each mobile node is assigned a speed of $110 \mathrm{~km} / \mathrm{h}$. Each communication scenario comprises of five mobile nodes (indexed as $0,1,2,3$, and 4). Here, host $_{0}$ and host $_{3}$ are within the communication range of each other as well as in the range of host $_{1}$. Similarly, host 2 and host 4 are within the communication range of each other as well as of 
host $_{1}$, as shown in Fig. 5.

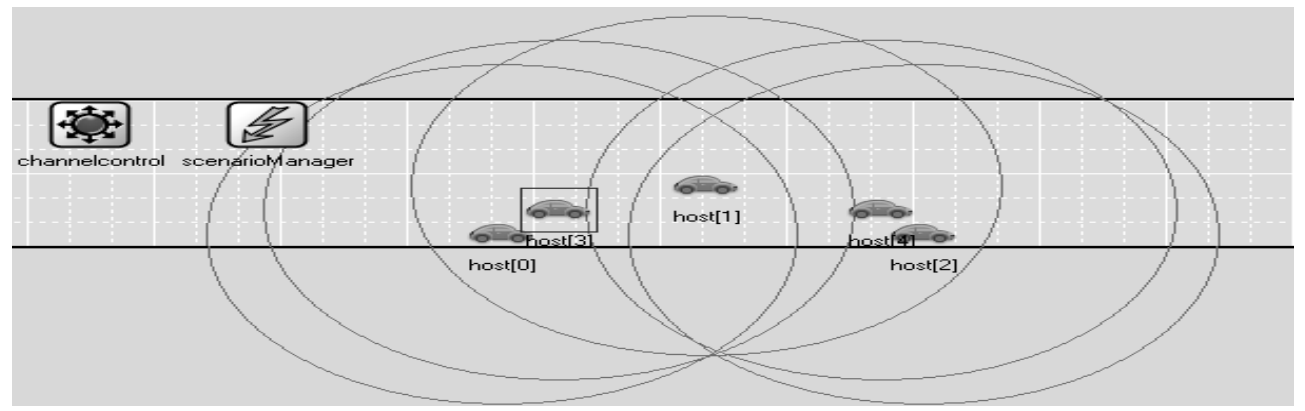

Fig. 5. VANET simulation layout in OMNeT++

It is assumed that each node has knowledge of its own location as well as of its one-hop neighboring nodes. Each packet contains 1500 bytes of data and duplicate packet transmission is assumed due to the packet drop in all scenarios.

In the first scenario, host $_{0}$ and host $_{2}$ transmit data packets to each other without coding at mobile relay node host ${ }_{1}$. In the second scenario, results are obtained while host $t_{0}$ and host $_{2}$ are involved in transmitting data packets to each other with coding that takes place at host ${ }_{1}$ and decoded at host $t_{0}$ and host $_{2}$. The third scenario depicts a situation, where before the transmission of actual data packets, host $_{0}$ and host $_{2}$ send RREQ and RREP packets to find routes to each other. Host ${ }_{1}$ encompassing other mobile nodes within its transmission range is responsible to perform the encoding of data packets (by using the XOR operation) in the second and the third scenarios, which depict hop-by-hop and source NC-Aware routing of data packets, respectively. Decoding of data packets takes place at respective destinations based on the XOR operation.

Different metrics (i.e., coding gain, and percentage of encoded transmissions) are used to analyze the performance of generalized source and hop-by-hop NC-Aware routing. Coding gain represents the ratio of the number of transmissions used by the non-coding approach to the number of transmissions used by the coding approach for the delivery of the same number of native (original) data packets. On the other hand, the percentage of the encoded transmissions can be defined as the total number of the encoded transmissions to total transmissions used to transfer a certain number of data packets within a wireless ad hoc networks. Simulations are performed by using linear mobility patterns of the INET framework [28] with uniformly distributed packet generation rates. By using different seed values, each scenario is executed ten times and the mean value is shown in Fig. 6 and Fig. 7.

Fig. 6 presents a comparison of source and hop-by-hop NC-Aware routing with a different number of the native data packets transmitted from both the sources host and $_{0}$ host $_{2}$ in terms of coding gain. Simulation results reveal that the overall coding gain with hop-by-hop NC-Aware routing approach remains higher than that of the source NC-Aware routing approach. Although, the coding gain for hop-by-hop approach remains higher than the source NC-Aware routing; however, the coding gain differential of these schemes varies with a packet drop rate. The packet drop ultimately results in an increased number of transmissions in the source routing due to the higher transmissions of control packets. 


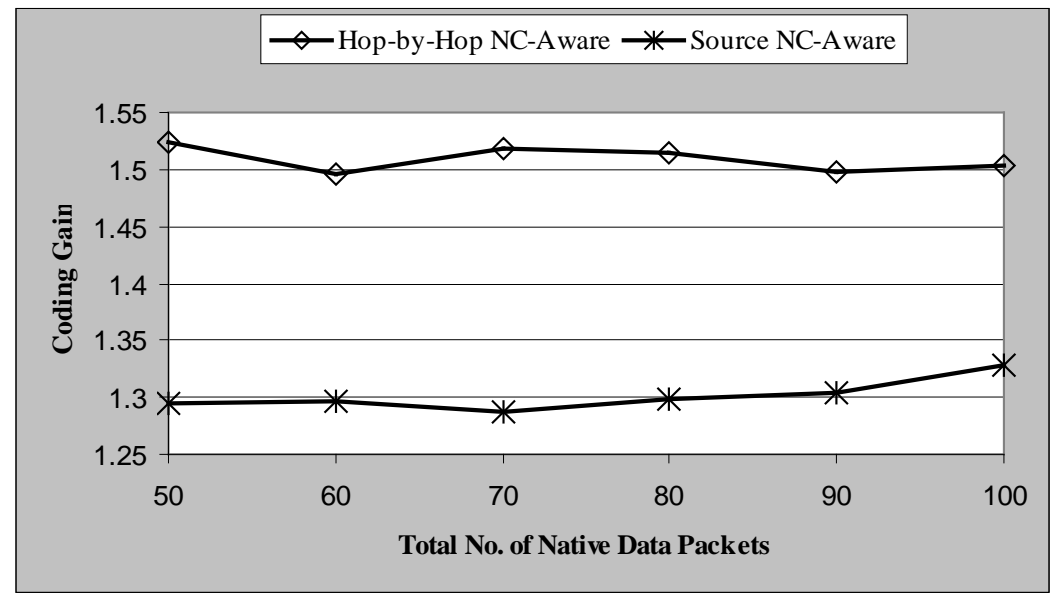

Fig. 6. Coding gain (source vs. hop-by-hop inter-flow coding approach)

Fig. 7 shows the percentage of the encoded transmissions over the total number of transmissions with source and hop-by-hop NC-Aware routing approach. Fig. 7 presents an evidence that the hop-by-hop NC-Aware routing approach is able to generate more coded transmissions. Therefore, the hop-by-hop technique outperforms source NC-Aware routing technique in terms of high data transfer towards the respective destinations.

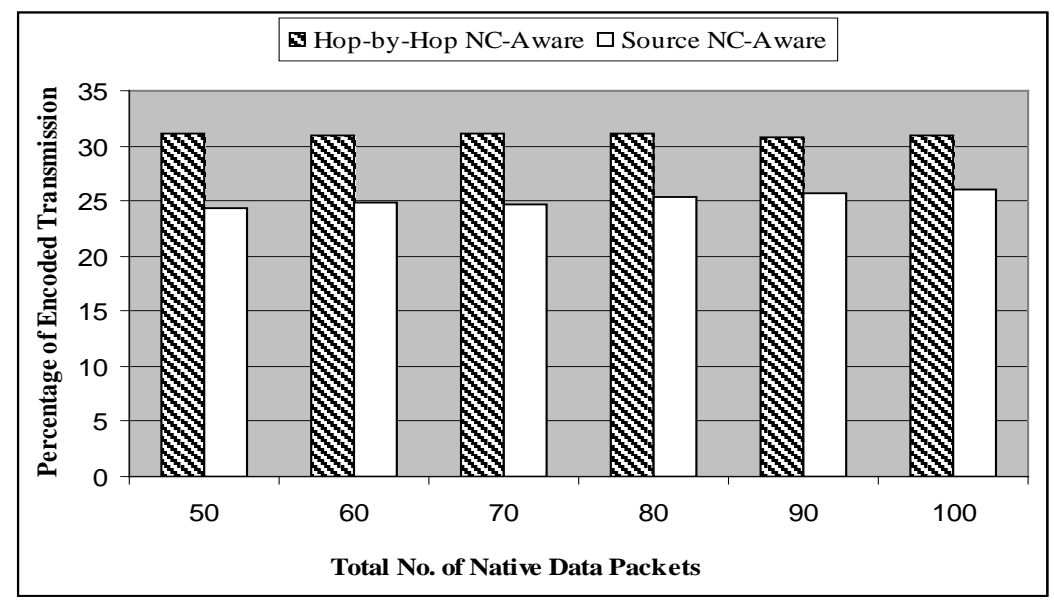

Fig. 7. Encoded packets percentage (source vs hop-by-hop NC-aware approach)

\section{Proposed Adaptive NC-Aware Routing Approach}

This section presents our proposed approach of adaptive coding-aware routing for data dissemination in a vehicular platoon. The proposed approach performs hop-by-hop inter-flow coding before forwarding packets towards destination vehicles while utilizing the fully broadcast nature of the wireless channel. Formal description of the implied notations and coding rules for the proposed adaptive NC-Aware routing scheme are illustrated below.

Concerning notations, graph $G=(V, E)$ represents VANET topology comprises of a set of vehicles $V$ and directed edges $E$ representing direct communication flows of vehicles within defined communication range. For a vehicle $V \in V$, subsets of data flow set $F$ denoted as $F$ (V) 
and $F^{+}(l)$ represents incoming and outgoing data flows, respectively. $U_{p}(V, F)$ and $D_{n}(V, F)$ denote the set of all upstream and downstream vehicles of vehicle $V$ on data flow path $F$, respectively. In addition, for coding rule descriptions, let $N(l)$ denotes the set of one-hop neighbors of $v$. At any instant of time, there may exist multiple data flows from vehicle $v_{i}$ to $V_{j}$ (i.e., $\left.F\left(V_{i}, V_{j}\right)\right)$ and vice verse (i.e., $\left.F^{+}\left(V_{i}, V_{j}\right)\right)$. Upon receiving multiple packets (i.e. $p_{i}, p_{i}^{-}, p_{j}, p_{j}^{-}$) belonging to opposite direction data flows (i.e. $\left.F\left(V_{i}\right), F^{+}\left(V_{i}\right), F\left(V_{j}\right), F^{+}\left(V_{j}\right)\right)$, respectively, the relay vehicle $V_{r}$ is able to encode all the received packets (i.e. $p=p_{i} \otimes p_{i}^{-} \otimes p_{j} \otimes p_{j}^{-}$) if

- $y_{r} \in$ Forwarder Selected Set of data flows of vehicles $V_{i}$ and $V_{j}$ and

- $N\left(V_{r}\right)=U_{p}\left(V_{i}, F^{-}\right) \cup D_{n}\left(V_{i}, F^{+}\right)$and $U_{p}\left(V_{j}, F^{-}\right) \cup D_{n}\left(V_{j}, F^{+}\right)$and

- $E\left(V_{r}\right)=E\left(V_{i}\right) \cup E\left(V_{j}\right)$

Considering the above coding rules, the design of the proposed adaptive coding-aware routing approach consists of the following components:

- Forwarder node selection

- Network topology estimation and orientation of multiple bidirectional data flows

- Encoding of packets in terms of availability of partial and impartial coding opportunities

- Forwarding of coded packets

For forwarder node selection, it is assumed that each node knows its own position through GPS and while taking into account the geo-distance towards the destination node, source nodes include the address of the destination node along with its own address and position. Therefore, based on this assumption, each broadcasted data packet carries the address as well as the location of the source node and the address of the destination node only. The address and location of the source node are required for the generation and broadcasting of the acknowledgment packet. The second component of the proposed approach deals with the estimation of the current flow-oriented topology of the network. After receiving packets from various sources, forwarder node can approximate the flow-oriented topology within the platoon by monitoring the address of the sender and the destination nodes. Another related step at this phase is opportunistic coding calculation that is the application of localized encoding of received native data packets from different flows on the basis of gained information about the topology and the flow of data packets from the source to the destination vehicles. Fundamentally, this step is related to the calculation of available coding opportunities and the appropriate encoding of data packets belong to different flows (that maximizes the coding gain), at the instance of forwarding time. The last component is the forwarding of encoded data packets towards the destination. However, before forwarding, coding node extracts and encodes data from selected native packets as well as the source ID of each source. The inclusion of the source ID in encoded data packets is required to generate acknowledgment against the successful decoding of a required data packet for that particular node. This hop-by-hop approach allows the coding nodes to opportunistically encode data packets after estimating the topology and flow of the data packets at any instance of time. Therefore, with the adaptive forwarding of maximum possible information in a single transmission, the proposed approach can significantly improve the network throughput. 


\subsection{Illustration of the Proposed Approach}

Consider a scenario in which FMM consisting of three lanes and five vehicles (A, B, C, D, and E) involved in platoon conversation moving in different lanes and there exist four communication flows (i.e. $f_{1}$ (from A to E), $f_{2}$ (E to A), $f_{3}$ (B to D), and $f_{4}$ (from D to B)) passing through a relay node $\mathrm{C}$, as shown in Fig. 8. At any instant of time $t_{1}$, it is considered that vehicles $A, B, D$, and $E$ are within the transmission range of vehicle $C$. In highway scenario, vertically it is obvious that vehicles A,B and D,E are within the communication range of each other. In the scenario shown in Fig. 8, vehicle $C$ is able to encode native data packets from different flows in various combinations. However, while estimating the topology of the network, only encoding combination of the native data packets $b_{1}, b_{4}$ and $b_{2}, b_{3}$ can significantly improve the performance of the network in this situation.

On the other hand, considering the same data flows but with the topology of Fig. 9 at any other instance of time $t_{2}$, vehicle $\mathrm{C}$ is able to encode all received native data packets within its queue and can further improve the performance of the network. The proposed approach consists of two major components i.e., detection of topology and communicating flows from received native data packets and accordingly do the appropriate encoding of native data packets through the calculation of beneficial coding opportunities. Details of the simulation modeling and obtained simulation results of the proposed scheme are discussed in section 4 and section 5 , respectively.

Informal description, upon receiving data packets, vehicle $C$ estimates that $U_{p}\left(C, F^{-}\right) U$ $D_{n}\left(C, F^{+}\right)=\{A, B, C, D\}$ and it also fulfills the condition i.e., $E(C)=E(A) U E(B) U E(D) U$ $E(E)$.

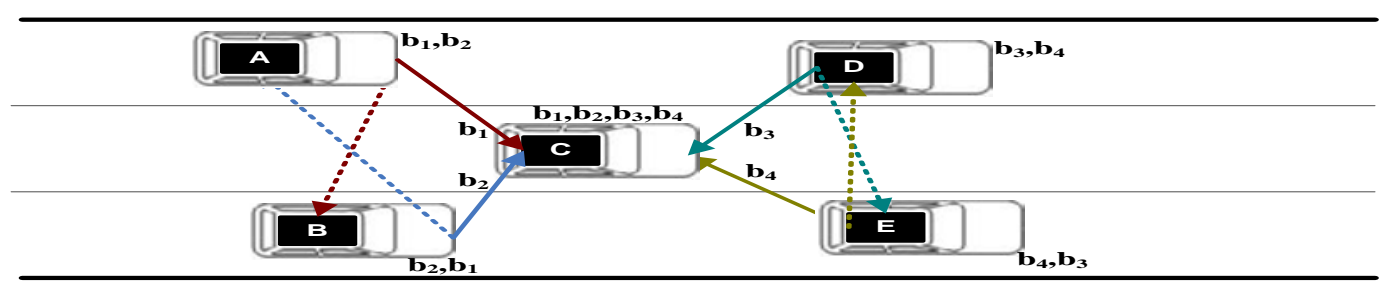

Fig. 8. Encoding of $b_{1}, b_{4}$, and $b_{2}, b_{3}$ at Node $C$ is Appropriate

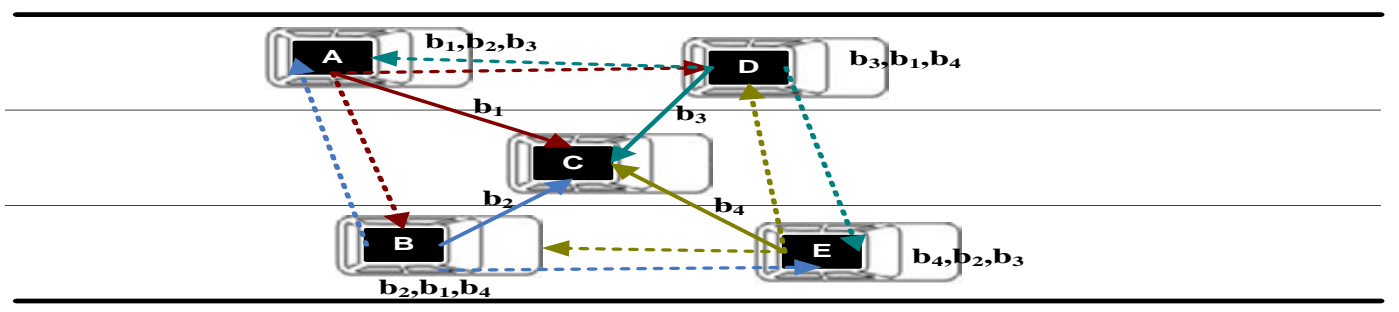

Fig. 9. Encoding of data packet from all data flows at coding node C

\section{Simulation Modeling}

In VANETs, economic issues, logistic complexities as well as the technological restraints have made simulations a prominent choice for the protocol evaluations. However, one of the primary challenges in VANET simulations is the realistic description of the vehicular mobility [29] [30]. In this work, the evaluation of the proposed adaptive NC-Aware routing protocol is performed in OMNeT++ by using modified mobility scenario of FMM, which is a depiction of 
a real highway scenario. Mathematical details of the modified FMM are discussed in Section 4.1.

\subsection{Modeling of the Modified FMM}

During the last decade, a number of realistic mobility models have been introduced to support the accurate evaluation of routing protocols for the real VANETs [29] [30]. In the same context, this work is concerned to evaluate the performance sensitivity of the NC-Aware routing in the modified mobility scenario of FMM. To define realistic mobility behavior of vehicles within the modified FMM, the notational method as described in [31], [32] is followed. For vehicle $N$, the terms $X_{n}(t), V_{n}(t)$ (or $V_{n}$ ), and $\Theta_{n}(t)$ are used to indicate the position, speed, and bumper to bumper distance respectively, at any time instance $t$, as shown in Fig. 10. The index $N \pm 1$ refers to the front and back vehicles following the same lane (in which $N$ is moving).

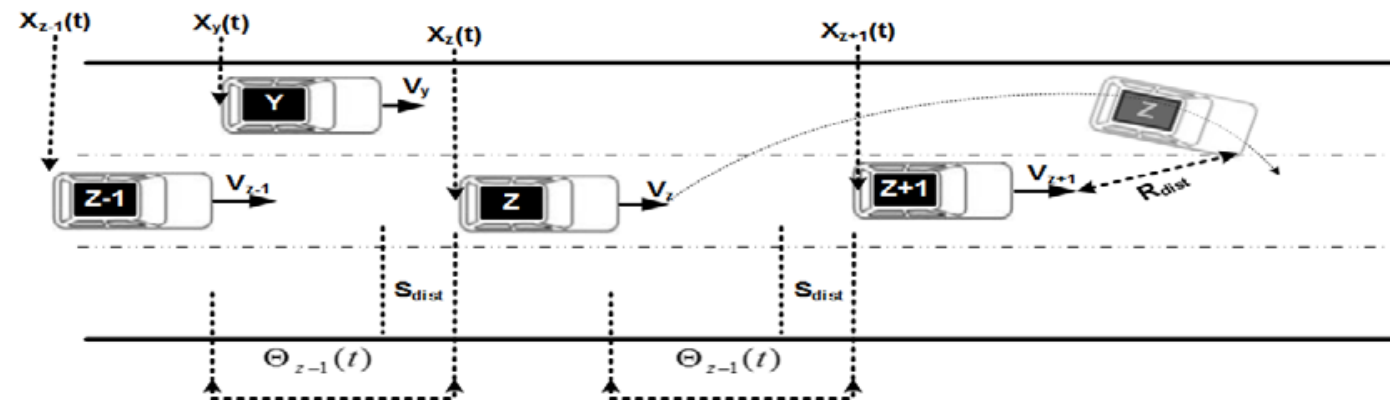

Fig. 10. Illustration of mobility notations

\subsubsection{Proposed Modified FMM}

FMM (proposed in [27]) is a generated-map-based model that depicts highway traffic. However, it is conceived as unrealistic due to its limitation in terms of overtaking and lane changing. To evaluate the performance of routing protocols with more realistic mobility patterns, FMM with certain modifications [33] has been implemented. While dealing as a category of car-following model, main features associated with the modified FMM are explained below:

- The simulation area comprises a number of freeways and ultimately each side of a freeway consists of three lanes i.e. fast, slow, and overtaking lane.

- Considering Fig. 10 and microscopic mobility description of [27], at any instance of time $t$, the speed of a vehicle $Z$ is confined to a lane is $V_{z}(t)$, and at each next time step $\Delta t$, the speed of this particular vehicle will be

$$
V_{z}(t+\Delta t)=V_{z}(t)+\eta \cdot a_{z}(t)
$$

where $a_{z}(t)$ is acceleration of vehicle $Z$ at any instance of time $t$, and the random variable $\eta$ is used to introduce randomness (in the range [-1,1]) while vehicles are updating their speed. For each vehicle, $V_{z}(t)$ is required to be within the range of maximum and minimum allowed speed as $\left[V_{\min }, V_{\max }\right] . \Delta V_{z}$ indicates a change in the speed of vehicle $Z$ and it must be

$$
\Delta V_{z}=\left|V_{z}(t)-V_{z}(t+\Delta t)\right| \quad \ldots \quad \text { (2) }
$$

It indicates that variation in the speed $\left(\Delta V_{z}\right)$ of a vehicle $Z$ is non-uniformly distributed within the interval [ $a_{\min } x \Delta t, a_{\max } x \Delta t$ ]. Here, for vehicle $Z$ at any instance of time, $a_{\min }$ and $a_{\max }$ represent allowed minimum and maximum deceleration/acceleration, respectively.

- While maintaining a safety distance $\left(S_{\text {dist }}\right)$ between subsequent vehicles, any two vehicles 
(say $Z$ and $Z+1$ of Fig. 10) are supposed to remain in a lane.

$$
\begin{gathered}
\forall_{z} \forall_{t} \quad \text { if } \Theta_{z}(t) \leq S_{\text {dist }} \text { then } \\
V_{z}(t) \leq V_{z+1}(t) \\
\frac{d V_{z}(t)}{d t} \leq 0
\end{gathered}
$$

Here, $\Theta_{z}(t)$ is the bumper-to-bumper distance of vehicle $Z$ from its anterior vehicle $Z+1$ (shown in Fig. 10), and the term $\frac{d V_{z}(t)}{d t}$ indicates the acceleration (of vehicle $Z$ ), at any instance of time $t$. Equation (3) points out the condition that if two vehicles come close to each other in a way that the bumper-to-bumper distance between them approaches $S_{\text {dist }}$ from each other. For this reason, while considering the vehicles $Z$ and $Z+1$ of Fig. 10 within the safety distance of each other, the condition states that within the $S_{\text {dist }}$, the speed of the succeeding vehicle $(Z)$ must not exceed the speed of the preceding vehicle $(Z+1)$. Therefore, to restrict overtaking, succeeding vehicle $(Z)$ is required to move either with constant speed or decelerate.

- Taking into account the Intelligent Driver Model with Lane Changing (IDM-LC) [31] [32], overtaking has been allowed in modified mobility models. A vehicle is allowed to overtake if the feasibility of overtaking vehicle (in terms of acceleration) is higher than the feasibility (in terms of deceleration) of coming vehicle from backside in the new lane (e.g. vehicle $Z$ and $Y$ in case of Fig. 10):

$$
\left(\frac{d V_{z}^{\prime}(t)}{d t}-\frac{d V_{z}(t)}{d t}\right) \geq\left(\frac{d V_{y}(t)}{d t}-\frac{d V_{y}^{\prime}(t)}{d t}\right) \quad \ldots
$$

Here $\frac{d V_{z}^{\prime}(t)}{d t}$ and $\frac{d V_{y}^{\prime}(t)}{d t}$ are used to specify updated acceleration and deceleration of vehicles $Z$ and $Y$ respectively, after lane changing. Moreover, it is required that sudden deceleration $\frac{d V_{y}^{\prime}(t)}{d t}$ deceleration) and at the same time, the sudden increase in vehicle $Z$ 's acceleration should be less than $a_{\max }$ as equation (5) points out.

$$
\frac{d V_{Z}^{\prime}(t)}{d t}<a_{\max }, \frac{d V_{y}^{\prime}(t)}{d t}>-a_{\text {min }}
$$

- For returning back to the previous lane, it is required for vehicle $Z$ to consider the acceleration as well as the distance of the posterior vehicle $Z+1$ in the previous lane. While entering into the previous lane, vehicle $Z$ considers the returning point distance $\left(R_{\text {dist }}\right)$ between itself and vehicle $Z+1$, which should be at least double than the $S_{\text {dist }}$ between these two vehicles. Furthermore, the change in acceleration of vehicle $Z$ is required to be greater than change of vehicle $(Z+1)$ 's acceleration.

$$
\left|\frac{d V_{z}^{\prime}(t)}{d t}-\frac{d V_{z}(t)}{d t}\right| \geq\left|\frac{d V_{z+1}(t)}{d t}-\frac{d V_{z+1}^{\prime}(t)}{d t}\right|
$$

The next step deals with the simulation modeling of modified FMM with the initial deployment of nodes at random positions in specified lanes of the defined simulation area. 


\subsubsection{Simulation Model for Adaptive NC-Aware Routing}

OMNeT++ with the available support of the INET framework is utilized for the performance evaluation of the proposed scheme. Various mobile nodes are deployed as compound modules (composed of various sub-modules i.e. mobility, pathfinding, and inter-flow coding application module etc.) in the simulation area. The overall simulation consists of various components defined within files of different extensions (i.e., .ned for network definitions, .cc for implementation definition with $\mathrm{C}++$, and .msg for message definitions). By taking into account various parameters (i.e., topology size, mobility directions, number of lanes etc.), mobile nodes following linear mobility model of the INET framework are considered for the road topography of three lanes with a length of $10 \mathrm{~km}$ and a width of $10 \mathrm{~m}$ per lane. The mobility details (at mobility layer) include the speed, acceleration, as well as coordinate positions of a particular node at any instance of time within the defined simulation area. The ChannelControl module of the INET framework is helpful in the provisioning of information related to the mobility and position of nodes within the defined. Moreover, for a particular mobile node, the ChannelControl module is used to discover other mobile nodes within its communication range. Another important module is the NotificationBoard that is required for the notification of events related to the change in the position of mobile nodes during the simulation. Implemented network coding module is responsible for the generation, encoding, and decoding of data packets. Moreover, this module is also concerned with the maintenance of Fist-In First-Out (FIFO) for virtual queues of a mobile node to store information of received packets and its neighboring nodes. For initial deployment of nodes, separately generated files containing details of initial coordinates of each mobile node is important to be included in the configuration files. Furthermore, for the configuration of each scenario, various parameters (i.e., dimensions of simulation area, the power to transmit packet/transmission range, and type of mobility with specified mobility parameters) are required to be set through specific .ini files. General parameters that have been considered for the implementation and evaluation of the NC-Aware routing are summarized in Table 1 and a screenshot of the defined simulation topology is shown in Fig. 11.

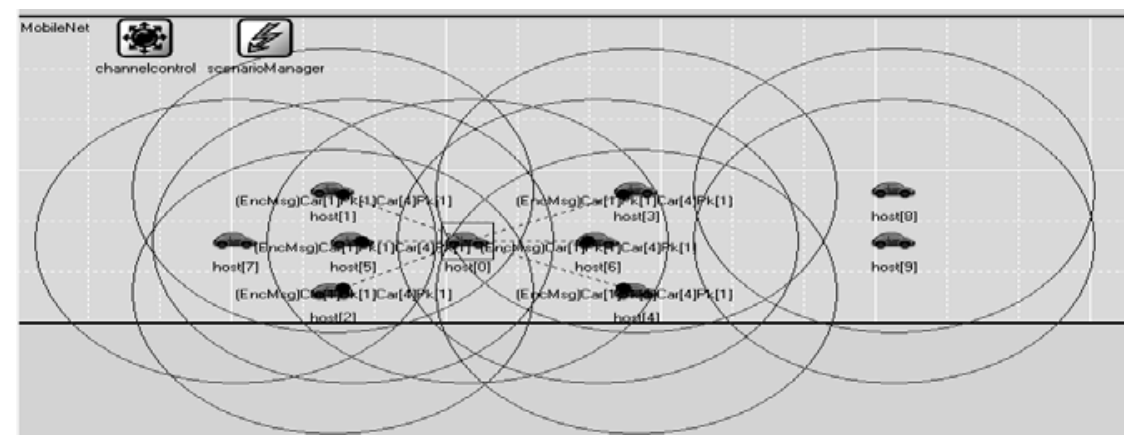

Fig. 11. Simulation screenshot of coding-aware data dissemination in OMNeT++

The last step is concerned with the definition of data packets. Each data packet contains information about the transmission power, channel number, coordinates of sender node, source/destination addresses, and the packet payload. 
Table 1. Simulation parameters

\begin{tabular}{|l|l|}
\hline \multicolumn{1}{|c|}{ Parameter } & \multicolumn{1}{c|}{ Value } \\
\hline Simulation Tool & OMNeT++ \\
\hline Simulation Time & 300-900 Sec. \\
\hline Mobility Model & Modified FMM \\
\hline Topology Size & $10 \mathrm{Km} \mathrm{x} \mathrm{60} \mathrm{m}$ \\
\hline Number of Lanes & 3 \\
\hline Lane width & $10 \mathrm{~m}$ \\
\hline Node Density & 10 \\
\hline Source nodes (Data Flows) & 4 \\
\hline Node Speed & $60 \mathrm{Km} / \mathrm{h}-120 \mathrm{Km} / \mathrm{h}$ \\
\hline Packet Size & $1500 \mathrm{bytes}$ \\
\hline Transmission Range & $250 \mathrm{~m}$ \\
\hline $\begin{array}{l}\text { Signal Attenuation } \\
\text { Threshold }\end{array}$ & $-110 \mathrm{dBm}$ \\
\hline
\end{tabular}

\section{Performance Evaluation of the Adaptive NC-Aware VANET Routing}

The simulation results of the proposed NC-Aware routing scheme in comparison with general hop-by-hop routing algorithm without coding (WNC) as well as with two (NC2) and four (NC4) packets coding-aware routing schemes are discussed in terms of average throughput, the percentage of encoded transmissions, and transmission percentage.

Average throughput is the amount of successfully received data by the receiver nodes per unit time and is calculated as the total number of bits received at each node divided by the simulation time. Percentage of the encoded transmissions shows the efficiency of a system and is calculated as the ratio of coded transmissions to the total number of transmissions within the network. Moreover, transmission percentage of different encoded packets within the proposed scheme is harnessed to describe the adaptability behavior between different encoding schemes within a particular scenario.

Graph curves of Fig. 12 indicate that the proposed adaptive coding scheme can significantly improve the network throughput as compared to the WNC scheme wherein the relay nodes forward data packets without encoding. 


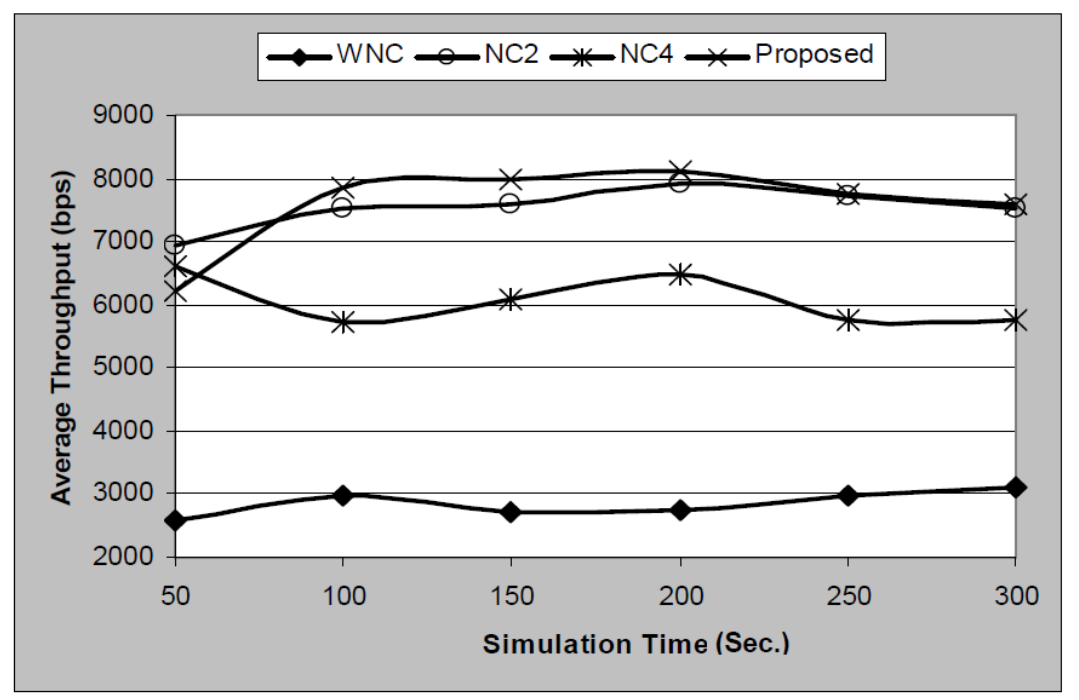

Fig. 12. Average throughput (proposed scheme with coding and non-coding approach)

Similarly, it is also noticed that average throughput for NC-Aware routing scenario of NC4 remains significantly lower in comparison with NC2 and the proposed approach. It is due to the reason that relay nodes have to wait a long time for the reception of data packets from four different flows to encode them together. Meanwhile, rapid change in topology lowers the possibility of encoding. However, the probability of rapid topology change is very low in a real vehicular platoon scenario.

Throughput differential of NC2 and NC4 indicates that encoding of data packets from two different flows is much higher in comparison with the encoding of four data packets from different flows. However, this throughput differential is highly dependent upon scenario or varies from scenario to scenario. The probability of four data packets encoding can be comparable to the probability of the encoding of two data packets in a highly congestion situation with a higher number of data flows. On the other hand, the proposed scheme also shows higher throughput than NC2. The throughput differential of the NC2 and the proposed scheme is not very high but the adaptive behavior supports the implication of proposed scheme in dynamic ad hoc networks. This is due to the fact that network topology is dynamic and with the information of current topology and the composition of multiple unicast data flows, the proposed approach is able to encode data packets with the actual coding opportunities available at coding nodes at any instance of time.

Fig. 13 shows that overall percentage of the encoded transmissions remains high for NC2. However, the proposed scheme with less percentage of the encoded transmissions is able to increase the throughput of the network as shown in Fig. 12. In general, a higher percentage of the encoded data packets leads to the higher network throughput; however, the adaptive nature of the proposed scheme improves the network throughput by maximizing the number of packets to be encoded in a single transmission. Therefore, while estimating the topology through the composition of data flows within the ad hoc scenario, proposed scheme connects localized encoding of maximum packets with hop-by-hop opportunistic forwarding of the data packets. The difference between the percentages of the encoded packet transmissions varies from scenario to scenario. It becomes evident from Fig. 13 that the encoded transmission differential varies during the simulation evaluation of a particular scenario. It is due to the reason that encoding probability of maximum data packets changes at every instance of time in 
dynamic ad hoc networks. Moreover, it is observed that the percentage of the NC4 coding scheme remains lower than that of NC2 and the proposed scheme and is the main cause of low network throughput. It is essential to specify that in a particular situation, NC4 scheme with a high probability of the encoding of four data packets from different flows can outperform NC2 at a low percentage of the encoded transmissions.

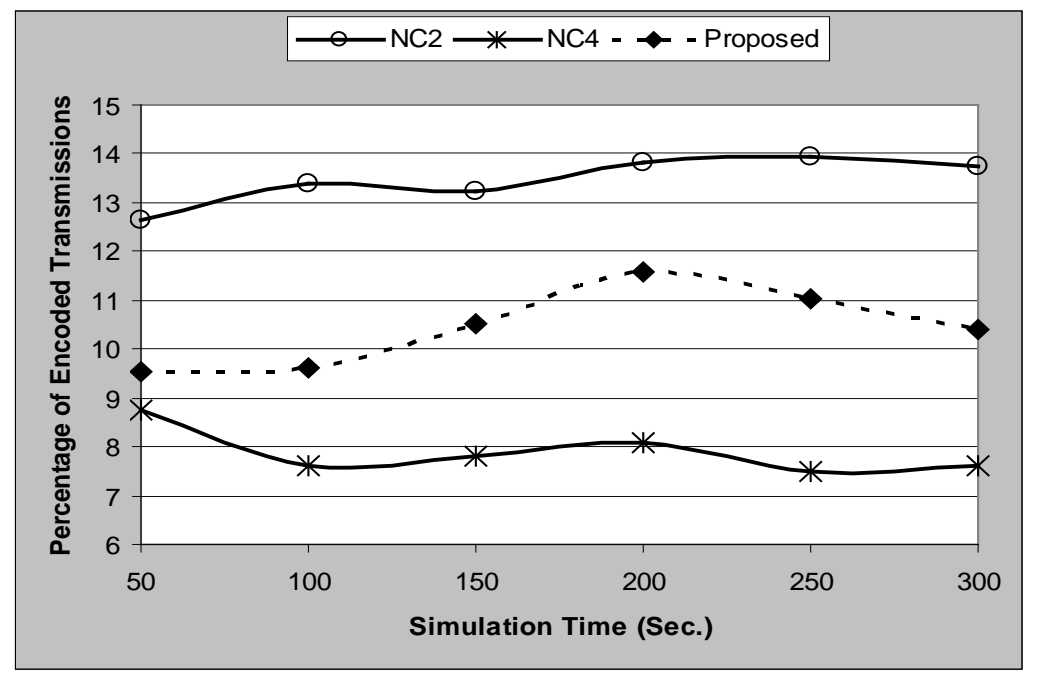

Fig. 13. Encoded transmission percentage (proposed scheme vs NC2 and NC4)

Fig. 14 indicates the transmission percentage of uncoded, two coded and four coded packets during the simulation. The changing trends of curves for two encoded and four encoded packet transmissions indicate the adaptability of the proposed scheme in coding packets according to the suitable opportunities.

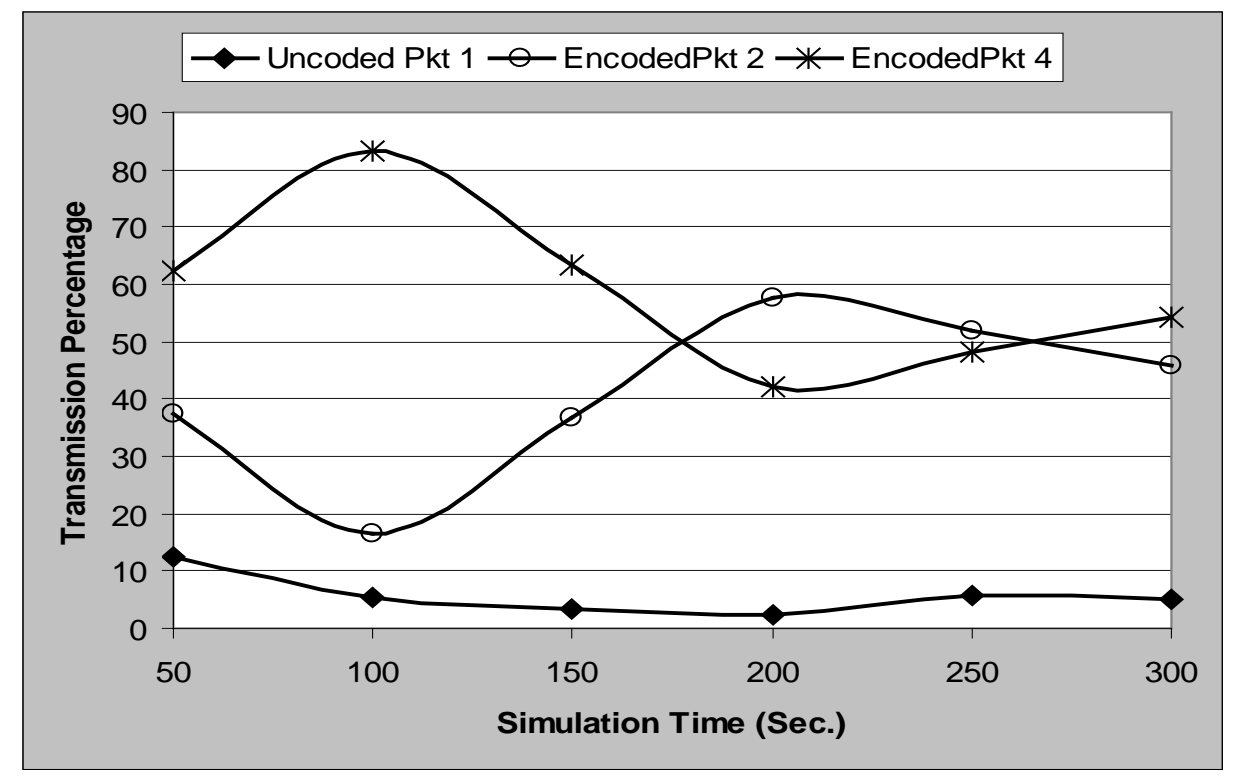

Fig. 14. Transmission percentage for un-coded, two- and four- coded packets in the proposed scheme 
Initially, at the start of the simulation, the proposed scheme adopted to encode four data packets more than two data packets (as shown in Fig. 13), and this is the main reason for the low percentage of the encoded transmissions at the start of simulation (as shown in Fig. 13). However, as the simulation proceeds, the proposed approach adopted to encode two packets (that is more frequent) and consequently high percentage of encoded transmissions can be seen in Fig. 13.

\section{Conclusions and Future Work}

Pertaining to the progress in the field of information theory and its association with VANETs, this study provides a fundamental basis for the application of the state-of-the-art NC-Aware data dissemination within VANETs in the context of routing. In order to comprehensively exploit the use of information related to the topology and the composition of unicast data flows within the VANETs, an adaptive topology-based flow-oriented NC-Aware routing scheme is proposed, which supports the dynamic encoding of data packets from particular data flows to improve the network performance. This work provides significant insights in support of the NC-Aware routing protocols in VANET scenario. The possible future research directions related to the implications of this area are listed below:

- Vehicular platoons can be of large-scale and therefore NC-Aware routing protocols should consider the appropriate level of scalability related to the encoding and decoding of data packets;

- From the discussion of NC-Aware routing protocols, it becomes evident that these protocols improve QoS parameters (i.e., throughput, reliability etc.) but there is a need of mechanism which is more concerned with the constraints within QoS parameters;

- The effect of dynamic behavior and design of light-weight NC-Aware routing approaches for low processing delays at each node are other future dimensions related to their implementation in dynamic ad hoc networks.

\section{References}

[1] C. Campolo, A. Molinaro, and R. Scopigno, "Vehicular Ad Hoc Networks: Standards, Solutions, and Research," Springer, 2015. Article (CrossRef Link)

[2] Y. Wang, D. Tian, Z. Sheng, and W. Jian, "Connected Vehicle Systems: Communication, Data, and Control," Taylor \& Francis Group, 2017. [ISBN-13: 978-1138035874]

Article (CrossRef Link)

[3] S. Al-Sultan, M. M. Al-Doori, A. H. Al-Bayatti, and H. Zedan, "A Comprehensive Survey on Vehicular Ad Hoc Network," Journal of Network and Computer Applications, vol. 37, pp. 380-392, 2014. Article (CrossRef Link)

[4] P. Kavathekar and Y. Chen, "Vehicle Platooning: A Brief Survey and Categorization," in Proc. of ASME 2011 International Design Engineering Technical Conferences and Computers and Information in Engineering Conference, pp. 829-845, 2011.

Article (CrossRef Link)

[5] L. Hobert, "A Study on Platoon Formations and Reliable Communication in Vehicle Platoons," Master Thesis, University of Twente, 2012. Article (CrossRef Link)

[6] Y. Zheng, S. E. Li, J. Wang, D. Cao, and K. Li, "Stability and Scalability of Homogeneous Vehicular Platoon: Study on the Influence of Information Flow Topologies," IEEE Transactions on Intelligent Transportation Systems, vol. 17, pp. 14-26, 2016. Article (CrossRef Link)

[7] D. Jia, K. Lu, J. Wang, X. Zhang, and X. Shen, "A Survey on Platoon-based Vehicular Cyber-physical Systems," IEEE Communications Surveys \& Tutorials, vol. 18, pp. 263-284, 2015. Article (CrossRef Link)

[8] H. Hartenstein and K. Laberteaux, "VANET: Vehicular Applications and Inter-Networking Technologies," Wiley Online Library, 2010. Article (CrossRef Link) 
[9] C. Sommer, F. Dressler, "Vehicular Networking," Cambridge University Press, 2017. Article (CrossRef Link)

[10] "OMNeT++: Discrete Event Simulator," Article (CrossRef Link)

[11] T. Chamberlain, "Learning OMNeT++," Packt Publishing, 2013. ISBN-10: 1849697140 Article (CrossRef Link)

[12] C. Boldrini, K. Lee, M. Önen, J. Ott, and E. Pagani, "Opportunistic Networks," Computer Communications, vol. 48, no. 14, pp.1-4, 2014. Article (CrossRef Link)

[13] T. Javidi and E. Van Buhler, "Opportunistic Routing in Wireless Networks," Foundations and Trends in Networking, vol. 11, no. 1-2, pp. 1-137, 2016. Article (CrossRef Link)

[14] M. A. Iqbal, B. Dai, B. Huang, A. Hassan, and S. Yu, "Survey of Network Coding-Aware Routing Protocols in Wireless Networks," Journal of Network and Computer Applications, vol. 34, pp. 1956-1970, 2011. Article (CrossRef Link)

[15] C. Wu, S. Ohzahata, Y. Ji, and T. Kato, "Multi-hop Broadcasting in VANETs Integrating Intra-flow and Inter-flow Network Coding," in Proc. of IEEE 80th Vehicular Technology Conference (VTC), pp. 1-6, 2014. Article (CrossRef Link)

[16] F. Liu, C. Zhiyong, and X. Bin. "Data Dissemination with Network Coding in Two-way Vehicle-to-Vehicle Networks," IEEE Transactions on Vehicular Technology, vol. 65, no. 4, pp. 2445-2456, 2016. Article (CrossRef Link)

[17] Q. Wang, D. Xie, and X. Ji, "Network Codes-based Content-centric Transmission Control in VANET," in Proc. of International Conference on Connected Vehicles and Expo (ICCVE), pp. 157-162, 2015. Article (CrossRef Link)

[18] G.M.N. Ali, M.A. Rahman, P.H.J. Chong, and S.K Samantha, "On Efficient Data Dissemination Using Network Coding in Multi-RSU Vehicular Ad Hoc Networks," in Proc. of IEEE 83rd Conference on Vehicular Technology (VTC), pp. 1-5, 2016. Article (CrossRef Link)

[19] L. Juan, X. Yi, L. Zhen, and L. Renfa, "Network-Coding Based Multicast Routing in VANET," Journal of Computer Research and Development, vol. 9, pp. 8, 2011. Article (CrossRef Link)

[20] I. Achour, T. Bejaoui, and S. Tabbane, "Network Coding-based Dissemination Protocols for Inter-vehicle Communication," in Proc. of IEEE International Symposium on Networks, Computers and Communications, pp. 1-6, 2014. Article (CrossRef Link)

[21] S. Sengupta, S. Rayanchu, and S. Banerjee, "Network Coding-aware Routing in Wireless Networks," IEEE/ACM Transactions on Networking, vol. 18, pp. 1158-1170, 2010. Article (CrossRef Link)

[22]C. Lu, S. Xiao, and Y. Miao. "OQMCAR: An Enhanced Network Coding-aware Routing Algorithm Based on Queue State and Local Topology," KSII Transactions on Internet \& Information Systems, vol. 9, no. 8, pp. 2875-2893, 2015. Article (CrossRef Link)

[23]X. Shao, R. Wang, H. Huang, and L. Sun, "Load Balanced Coding-Aware Multipath Routing for Wireless Mesh Networks," Chinese Journal of Electronics, vol. 24, no. 1, pp.8-12, 2015. Article (CrossRef Link)

[24]Y. Gu, H. Han, X. Li,. and J. Guo, "Network Coding-aware Routing Protocol in Wireless Mesh Networks," Tsinghua Science and Technology, vol. 20, no. 1, pp.40-49, 2015. Article (CrossRef Link)

[25]L. Hai, J. Wang, P. Wang, H. Wang, and Y. Tingting, "High Throughput Network Coding-Aware Routing in Time-Varying Multi-Hop Networks," IEEE Transactions on Vehicular Technology, vol. 66, no. 7, 2017. Article (CrossRef Link)

[26] Z. Mei, and Y. Zhen, "Active Intersession Network Coding-aware Routing," Wireless Networks, vol. 23, no. 4, pp. 1161-1168, 2017. Article (CrossRef Link)

[27] F. Bai, N. Sadagopan, and A. Helmy, "The IMPORTANT Framework for Analyzing the Impact of Mobility on Performance of RouTing Protocols for Adhoc NeTworks," Ad Hoc Networks, vol. 1, pp. 383-403, 2003. Article (CrossRef Link)

[28] "INET Framework," Article (CrossRef Link)

[29] C. Celes, F. Silva, A. Boukerche, R. Andrade, and A. Loureiro, "Improving VANET Simulation with Calibrated Vehicular Mobility Traces," IEEE Transactions on Mobile Computing, 2017. Article (CrossRef Link) 
[30] S. Ahmed, S. and S. S. Kangere, "On the Characterisation of Vehicular Mobility in a Large-scale Public Transport Network," Int. Journal of Ad hoc and Ubiquitous Computing, vol. 11, no. 2/3, pp.68-81, 2012. Article (CrossRef Link)

[31] H. Hartenstein and K. Laberteaux, "VANET Vehicular Applications and Inter-Networking Technologies," West Sussex, UK: Wiley Online Library, 2010. Article (CrossRef Link)

[32] M. Fiore and J. Härri, "The Networking Shape of Vehicular Mobility," in Proc. of ACM MobiHoc'08, pp. 261-272, 2008. Article (CrossRef Link)

[33]M. A. Iqbal, F. Wang, X. Xu, S. M. Eljack, and A. H. Mohammad, "Reactive Routing Evaluation using Modified 802.11a with Realistic Vehicular Mobility," Annals of Telecommunications-annales Des Télécommunications, vol. 66, pp. 643-656, 2011. Article (CrossRef Link)

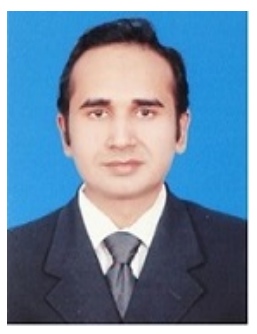

Muhammad Azhar Iqbal received PhD degree in Communication and Information Systems from Huazhong University of Science and Technology, Wuhan, P.R. China. His area of specialization is "Wireless Ad hoc Networks". Currently, he is working as Assistant Professor in the Department of Computer Science at Capital University of Science \& Technology, Islamabad. Moreover, he is also a member of Parallel Computing and Networks research group at Capital University of Science \& Technology, Islamabad. His research interests include the development and performance analysis of network coding aware routing and data dissemination techniques suitable to Vehicular Ad hoc Networks and Wireless Body Area Networks.

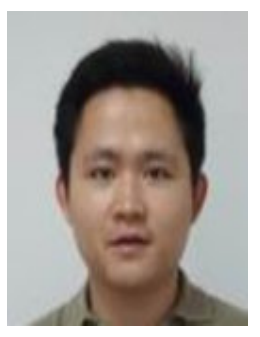

Bin Dai received his M. Eng. and PhD degrees from Huazhong University of Science and Technology (HUST), P. R. China in 2002 and 2006, respectively. From 2007 to 2008, he was a Research Fellow at the City University of Hong Kong. He is currently an Associate Professor at Department of Electronic Information and Communications, Huazhong University of Science and Technology, P. R. China. His research interests include P2P network, wireless network, network coding, and multicast routing.

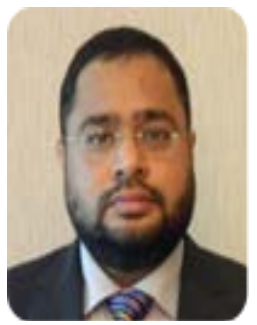

Muhammad Aleem received $\mathrm{PhD}$ degree in Computer Science from the Leopold-Franzens-Universität Innsbruck, Innsbruck, Austria in 2012. In August 2012, he joined Capital University of Science and Technology, Islamabad and currently working as Assistant Professor. His area of specialization is Parallel Computing. He is a member and co-founder of Parallel Computing and Networks research group at Capital University of Science \& Technology Islamabad. 


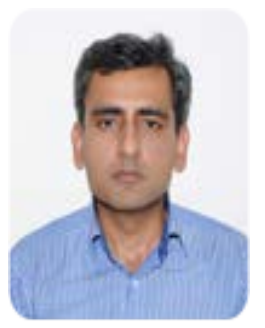

Muhammad Arshad Islam has been working at Capital University of Science \& Technology Islamabad as Assistant Professor in the department of computer science since February 2013. He is currently member of Parallel Computing and Networks research group at Capital University of Science \& Technology Islamabad. Mr. Islam has completed his PhD from University of Konstanz, Germany in May 2011 as DAAD scholar.

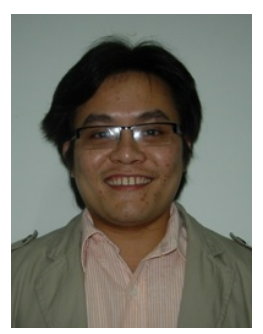

Nguyen-Son Vo received the B.Sc. degree in Electrical - Electronics Engineering and the M.Sc degree in Radio Engineering - Electronics from the Ho Chi Minh City University of Technology in 2002 and 2005, respectively. He then obtained his PhD degree in Communication and Information Systems from Huazhong University, China, in 2012. His current research interests include multimedia streaming over wireless networks, physical layer security, energy harvesting, and optimization design. 